\title{
Anomalous dimensions of spinning operators from conformal symmetry
}

\author{
Ferdinando Gliozzi \\ Dipartimento di Fisica, Università di Torino and INFN — Sezione di Torino, \\ Via P. Giuria 1, I-10125 Torino, Italy \\ E-mail: gliozzi@to.infn.it
}

ABSTRACT: We compute, to the first non-trivial order in the $\epsilon$-expansion of a perturbed scalar field theory, the anomalous dimensions of an infinite class of primary operators with arbitrary spin $\ell=0,1, \ldots$, including as a particular case the weakly broken higher-spin currents, using only constraints from conformal symmetry. Following the bootstrap philosophy, no reference is made to any Lagrangian, equations of motion or coupling constants. Even the space dimensions $d$ are left free. The interaction is implicitly turned on through the local operators by letting them acquire anomalous dimensions.

When matching certain four-point and five-point functions with the corresponding quantities of the free field theory in the $\epsilon \rightarrow 0$ limit, no free parameter remains. It turns out that only the expected discrete $d$ values are permitted and the ensuing anomalous dimensions reproduce known results for the weakly broken higher-spin currents and provide new results for the other spinning operators.

KeYwords: Conformal Field Theory, Higher Spin Symmetry

ARXiv EPrint: 1711.05530 


\section{Contents}

1 Introduction 1

2 Null states 4

$\begin{array}{lll}3 & \text { Spinning operators } & 7\end{array}$

$\begin{array}{lll}4 & \text { Matching conditions } & 10\end{array}$

5 Conclusions 13

$\begin{array}{ll}\text { A OPE coefficients of the free-field theory } & 15\end{array}$

\section{Introduction}

The conformal bootstrap approach [1-3], in its modern revival [4-36], is the latest of a set of powerful tools applied to the study of critical systems in different dimensions. One of the most surprising features of this approach is that some of the fundamental properies characterizing the critical systems, like the scaling dimensions of local operators and their operator product expansion (OPE) coefficients are (numerically) obtained under the only assumption that these systems are described by a conformal field theory (CFT), with no reference to Lagrangians, coupling constants or equations of motion. On the contrary in all the conventional approaches, like $\epsilon$-expansions [37] or Monte Carlo calculations, the choice of a specific Lagrangian is mandatory. This intriguing difference between conventional and bootstrap approaches calls for an analytical explanation.

A first step in this direction was taken in ref. [38] in the context of $\phi^{4}$ theory in $4-\epsilon$ dimensions. It was shown that the anomalous dimensions of scalar operators at the first non-trivial order in the $\epsilon$-expansion can be computed under the following three axioms: (I) The Wilson-Fisher (WF) fixed point is described by a CFT. (II) in the $\epsilon \rightarrow 0$ limit the vacuum expectation values of $n$-point functions approach those of the free field theory. (III) At the WF fixed point $\phi^{3}$ is a descendant of $\phi$ as a consequence of the equation of motion. Such an approach has been generalized in various ways in [39-46]. In particular in [43] it was shown that the axiom (III) is redundant and, in the spirit of bootstrap, no equations of motion nor any kind of Lagrangians were assumed. How to turn on the interaction without resorting to any interaction term or coupling constant? The recipe is very simple: one looks for a consistent smooth conformal deformation of a free theory in $d-\epsilon$ where the scaling dimensions $\Delta_{\mathcal{O}}$ of the operators $\mathcal{O}$ of the deformed theory differ from those $\mathcal{O}_{f}$ of the free theory with the same quantum numbers by corrections which can be expanded in powers of $\epsilon$

$$
\Delta_{\mathcal{O}}=\Delta_{\mathcal{O}_{f}}+\gamma_{\mathcal{O}}=\Delta_{\mathcal{O}_{f}}+\gamma_{\mathcal{O}}^{(1)} \epsilon+\gamma_{\mathcal{O}}^{(2)} \epsilon^{2}+\ldots
$$


where the $\gamma_{\mathcal{O}}$ 's are the anomalous dimensions. A stringent requirement is that there is a one-to-one mapping between the local operators of the free and the interacting theory. It turns out that this deformation can be consistently made only for some special values of the space-time dimensions $d$. These coincide with the upper critical dimension $d_{m}=$ $2 m /(m-2)$ (integer or rational [47]) where, in the standard RG approaches to a scalar theory perturbed with a $\phi^{m}$ potential, the WF fixed point is defined as a non-trivial zero of the $\beta$-function in $d=d_{m}-\epsilon$.

Matching suitable four-point functions in the $\epsilon \rightarrow 0$ limit with the free theory data yields at once the anomalous dimensions of $\phi^{p}$ for any integer $p$ at the first non-trivial order, while so far no information was obtained on the spectrum of spinning operators within such a method. There are other approaches that provide it for the special case of weakly broken higher-spin (HS) currents. For instance, from a Mellin space representation of the four-point, the anomalous dimensions of these currents and other scalar operators up to $\epsilon^{3}[48-50]$ and even higher order [51] have been obtained. Similarly, in a study relying in a standard Lagrangian description of conformal theories with weakly broken HS symmetry, the leading order anomalous dimensions of these currents have been determined from the classical non-conservation equations [52, 53]. WF fixed points with $O(N)$ symmetry have been studied in the large global charges limit in [54]. In another study relying instead in the conformal bootstrap [55, 56], the spectrum of these broken currents, at the leading order, follows from crossing symmetry and the exact conservation of the stress tensor.

The aim of this paper is to compute, at the leading order in $\epsilon$, the anomalous dimensions of a much larger class of primary operators $\mathcal{O}_{p, \ell}$ of arbitrary spin $\ell$, namely a set of those that in the Gaussian limit can be written in terms of symmetric traceless combinations of $\ell$ derivatives and $p+1$ copies of the scalar field $\phi$. Their scaling dimensions can be written as

$$
\Delta_{p, \ell}=(p+1) \frac{d-2}{2}+\ell+\gamma_{p, \ell},
$$

with $\gamma_{p, \ell}=\gamma_{p, \ell}^{(1)} \epsilon+\gamma_{p, \ell}^{(2)} \epsilon^{2}+\ldots$. The special case $p=1$ describes the HS conserved currents which are no longer conserved (for $\ell \neq 2$ ) at the WF fixed point, as they acquire an anomalous dimension; they are called sometimes weakly broken HS currents. On the contrary the stress tensor turns out to be exactly conserved in the present approach, as expected.

All our calculations rely in a unique group-theoretical mechanism, the conformal multiplet recombination first pointed out in [38]. Here we apply it in the form developed in $[43,45]$. Schematically, taking $d \sim 4$ for simplicity, the argument runs as follows. Consider the following fusion rule of the Gaussian theory

$$
\left[\mathcal{O}_{p, \ell}\right] \times\left[\phi_{f}^{p}\right]=\mathrm{c}_{1}\left[\phi_{f}\right]+\mathrm{c}_{2}\left[\phi_{f}^{3}\right]+\ldots,
$$

where $[\mathcal{O}]$ denotes a (suitably normalized) primary, the $c_{i}$ 's are OPE coefficients and the dots contain all other primaries which do not participate in the game. Using the equation above, the four-point function $\left\langle\mathcal{O}_{p, \ell} \phi_{f}^{p} \mathcal{O}_{p, \ell} \phi_{f}^{p}\right\rangle$ can be written as

$$
\left\langle\phi_{f}^{p} \mathcal{O}_{p, \ell} \mathcal{O}_{p, \ell} \phi_{f}^{p}\right\rangle=c_{1}^{2} \sum_{\alpha \in \mathcal{H}_{\phi_{f}}} \frac{\left\langle\phi_{f}^{p} \mathcal{O}_{p, \ell} \mid \alpha\right\rangle\left\langle\alpha \mid \mathcal{O}_{p, \ell} \phi_{f}^{p}\right\rangle}{\langle\alpha \mid \alpha\rangle}+c_{2}^{2} \sum_{\alpha \in \mathcal{H}_{\phi_{f}^{3}}} \frac{\left\langle\phi_{f}^{p} \mathcal{O}_{p, \ell} \mid \alpha\right\rangle\left\langle\alpha \mid \mathcal{O}_{p, \ell} \phi_{f}^{p}\right\rangle}{\langle\alpha \mid \alpha\rangle}+\ldots
$$


$\alpha \in \mathcal{H}_{\mathcal{O}}$ denotes a state of the conformal multiplet generated by the primary $\mathcal{O}|0\rangle=|\mathcal{O}\rangle$ and normalized so that $\langle\mathcal{O} \mid \mathcal{O}\rangle=1$. At the WF fixed point, because of the mentioned recombination, $\left[\phi^{3}\right]$ is absorbed by $[\phi]$ as a sub-representation and the fusion rule becomes

$$
\left[\mathcal{O}_{p, \ell}\right] \times\left[\phi^{p}\right]=\left(c_{1}+O(\epsilon)\right)[\phi]+\ldots
$$

Thus in the corresponding four-point function the second sum disappears while in the first sum new terms show up because now the conformal multiplet $[\phi]$ becomes long:

$$
\begin{aligned}
\left\langle\phi^{p} \mathcal{O}_{p, \ell} \mathcal{O}_{p, \ell} \phi^{p}\right\rangle=\left(c_{1}^{2}+O(\epsilon)\right)\left(\sum_{\alpha \in \mathcal{H}_{\phi_{f}}}\right. & \frac{\left\langle\phi_{f}^{p} \mathcal{O}_{p, \ell} \mid \alpha\right\rangle\left\langle\alpha \mid \mathcal{O}_{p, \ell} \phi_{f}^{p}\right\rangle}{\langle\alpha \mid \alpha\rangle} \\
& \left.+\sum_{\beta \in \mathcal{H}_{\chi}} \frac{\left\langle\phi^{p} \mathcal{O}_{p, \ell} \mid \beta\right\rangle\left\langle\beta \mid \mathcal{O}_{p, \ell} \phi^{p}\right\rangle}{\langle\beta \mid \beta\rangle}\right)+\ldots
\end{aligned}
$$

$\chi$ is a descendant of $\phi$ with scaling dimension $\Delta_{\chi}=\frac{d}{2}+1+O\left(\epsilon^{2}\right)$. The norm of $|\chi\rangle$ depends on that of $|\phi\rangle$ and goes to zero in the $\epsilon \rightarrow 0$ limit (for more details see next section). Actually the $c_{2}^{2}$ of the free theory is replaced by

$$
\mathrm{c}_{2}^{2} \rightarrow \mathrm{c}_{1}^{2} \frac{\left\langle\phi^{p} \mathcal{O}_{p, \ell} \mid \chi\right\rangle\left\langle\chi \mid \mathcal{O}_{p, \ell} \phi^{p}\right\rangle}{\langle\chi \mid \chi\rangle}
$$

In the $\epsilon \rightarrow 0$ limit $\chi$ is indistinguishable from $\phi_{f}^{3}$, hence the two expansions (1.4) and (1.6) must coincide. We find an infinite set of matching conditions, one for each pair of integers $p=1,2, \ldots$ and $\ell=0,1, \ldots$,

$$
\lim _{\epsilon \rightarrow 0} \frac{\left[\left(\gamma_{\phi}+\gamma_{\phi^{p}}-\gamma_{p, \ell}\right)\left(\gamma_{\phi}+\gamma_{\phi^{p}}-\gamma_{p, \ell}+2-d-2 \ell\right)\right]^{2}}{4 d(d-2) \gamma_{\phi}}=\left(\frac{c_{2}}{c_{1}}\right)^{2} .
$$

In this form such constraints are valid not only for $d$ near 4 , but also for $d$ near 3 , the upper critical dimension of a $\phi^{6}$ perturbation and, more generally, for the fractional $d=2 k /(k-1)$ corresponding to the upper critical dimension of the multicritical points of higher order, associated with $\phi^{2 k}$ potentials.

For $\ell=0$ the equations above coincide with those found in $[43,45]$ and used to compute $\gamma_{\phi^{p}}$ to the first order for any integer $p>1$ and to the second order for $p=1$. Here we extend the calculation to any spin $\ell$. In the particular case of weakly broken higher-spin currents, i.e. $p=1$, we have $\mathrm{c}_{2}=0$ and these matching conditions tell us simply that $\gamma_{1, \ell}^{(1)}=0$, a well known property of the WF fixed point at $d=4-\epsilon$ and at $d=3-\epsilon$. In order to calculate the first non-vanishing term of $\gamma_{\ell} \equiv \gamma_{1, \ell}$ we found it useful to study certain five-point functions which lead to the right answer in a surprisingly simple way. We found indeed the following new set of matching conditions

$$
\lim _{\epsilon \rightarrow 0} \frac{\left(\gamma_{\ell}-2 \gamma_{\phi}\right)\left(d-4+2 \ell+\gamma_{\ell}-2 \gamma_{\phi}\right)(d-2+2 \ell)}{2 d(d-2) \gamma_{\phi}}=m-1 .
$$

Here $m$ is an arbitrary integer $m>2$ and $d=d_{m}-\epsilon$, where

$$
d_{m}=\frac{2 m}{m-2}
$$


is the upper critical dimension for a perturbing potential $\phi^{m} .{ }^{1}$ In particular these matching conditions give at once, at the first non-vanishing order, the anomalous dimensions of the weakly broken HS currents for any $\ell$ in $d-\epsilon$ space-time dimensions with $d=3,4,6$. According to our definitions, $\gamma_{\ell=0} \equiv \gamma_{\phi^{2}}$. In such a case these equations allow to compute the $\epsilon^{2}$ contribution of $\gamma_{\phi^{2}}$ in the multicritical cases. This was not possible with the first set (1.8) of constraints.

Admittedly, owing to the huge amount of tensor structures involved, no much is known about the properties of the conformal block expansions of the four-point function (1.6) for general $\ell$. Much less is known about similar expansions of the five-point. However we are only interested in the couplings of the external operators to the scalar $\chi$. Here only a single tensor structure appears and all calculations simplify dramatically.

This paper is organized as follows. In section 2 we describe a simple algebraic method to classify all the possible representations of the conformal group in $d$ dimensions admitting a primary descendant in the case of symmetric traceless representations of the rotation subgroup $\mathrm{SO}(d)$. In section 3 we collect some useful properties of primary operators with spin, in section 4 we write the general matching conditions and their consequences. The body of the paper includes also some conclusions. Appendix A contains some formulas of the free field theory which are useful to compute the OPE coefficients $c_{1}$ and $c_{2}$.

\section{$2 \quad$ Null states}

The Euclidean conformal group $\mathrm{SO}(d+1,1)$ admits representations that are reducible but not completely reducible, i.e. they can have an invariant subspace whose complement is not invariant. The states of this invariant subspace have null norm (null states) and the corresponding multiplet is said to be long. The bosonic free field theory does not have this kind of representations (the corresponding conformal multiplets are short). The WilsonFisher fixed points can be seen as the points where some multiplets recombine to form a long multiplet. ${ }^{2}$

It is instructive to investigate the mechanism of appearance of null states operating with the Lie algebra of the conformal group. A standard basis is $D, P_{\mu}, K_{\mu}, J_{\mu \nu}$, $(\mu, \nu=1, \ldots d)$, which generate respectively the dilatation, the translations, the special conformal transformations and the $\mathrm{SO}(d)$ rotations. Applying this algebra to a state $|\Delta, \ell\rangle=\lim _{\epsilon \rightarrow 0} \mathcal{O}_{\Delta, \ell}(x)|0\rangle$, where $\mathcal{O}_{\Delta, \ell}$ is an arbitrary local operator and $|0\rangle$ a conformally invariant vacuum, generates a whole representation of the conformal group. A state with minimal $\Delta_{\min }=\Delta^{\prime}$ is said primary, all other states, where $\Delta=\Delta^{\prime}+n,(n=1,2 \ldots)$ are descendants. A primary state is annihilated by the $K_{\mu}$ 's. If $\left|\Delta^{\prime}, \ell^{\prime}\right\rangle$ is the only state which is annihilated by the $K_{\mu}$ 's the representation is irreducible, while if there is a primary descendant, i.e. a descendant state with $K_{\mu}\left|\Delta^{\prime}+n, \ell\right\rangle=0$, this state has null norm and generates an invariant subspace of null states.

\footnotetext{
${ }^{1}$ In most cases we have to assume $m$ to be an even integer and we find it useful to parameterize it with $m=2 q+2$, with $q=1,2, \ldots ; q$ and $d$ are related by $d=2(q+1) / q$.

${ }^{2}$ Actually this multiplet does not contain exactly null states, but states whose norm goes to zero as their scaling dimension approaches the free field value.
} 
The conformal multiplets generated by the fusion of two scalars correspond to symmetric, traceless tensors. The complete list of the primary descendants that could appear in the OPE of two scalars can be obtained in various ways. One point of attack follows form the study of the conformal blocks $G_{\Delta, \ell}\left(u=\frac{x_{12}^{2} x_{34}^{2}}{x_{13}^{2} x_{24}^{2}}, v=\frac{x_{23}^{2} x_{14}^{2}}{x_{13}^{2} x_{24}^{2}}\right)$ contributing to a four-point function of scalars $\left\langle\mathcal{O}_{1}\left(x_{1}\right) \mathcal{O}_{2}\left(x_{2}\right) \mathcal{O}_{3}\left(x_{3}\right) \mathcal{O}_{4}\left(x_{4}\right)\right\rangle$. These are eigenfunctions of the Casimir invariants $C_{2}, C_{4}, \ldots$ that can be formed with the Lie generators

$$
C_{i} G_{\Delta, \ell}(u, v)=c_{i}(\Delta, \ell) G_{\Delta, \ell}(u, v),
$$

where the first two Casimir eigenvalues are, respectively,

$$
\begin{aligned}
c_{2}(\Delta, \ell)= & \frac{1}{2} \Delta(\Delta-d)+\frac{1}{2} \ell(\ell+d-2), \\
c_{4}(\Delta, \ell)= & \Delta^{2}(\Delta-d)^{2}+\frac{1}{2} d(d-1) \Delta(\Delta-d) \\
& +\ell^{2}(\ell+d-2)^{2}+\frac{1}{2}(d-1)(d-4) \ell(\ell+d-2) .
\end{aligned}
$$

Higher order Casimir invariants are redundant when considering symmetric, traceless tensors. These conformal blocks can be written as sum of poles in $\Delta$. As eq. (1.6) suggests, poles occur at special $\Delta$ 's where a descendant state becomes null. The location of poles, their residues as well as the remaining entire function can be computed [20] solving the Casimir differential equations. Ref. [57] uses instead a group-theoretical method, looking for all primary descendants that arise when the dimension $\Delta^{\prime}$ of the primary varies. In both cases one finds three sequences of poles, as shown in table 1 .

Here we give a two-line algebraic derivation of such a table. Let $\left|\Delta^{\prime}, \ell^{\prime}\right\rangle$ be a primary state and $\left|\Delta=\Delta^{\prime}+n, \ell\right\rangle$ a primary descendant. Since they belong to the same representation, they must share the eigenvalues of the Casimir invariants, namely

$$
c_{2}\left(\Delta^{\prime}, \ell^{\prime}\right)=c_{2}(\Delta, \ell), c_{4}\left(\Delta^{\prime}, \ell^{\prime}\right)=c_{4}(\Delta, \ell) .
$$

As first pointed out in [45], the solutions of these two algebraic equations yield the complete list of primaries admitting a primary descendant, reproduced in table 1 . End of the proof. ${ }^{3}$

If $d$ is an even integer, some of the above solutions coalesce, giving rise to double poles in the conformal blocks [57]. In this paper we study theories in $d-\epsilon$ dimensions, so we do not need to consider these exceptional cases.

Among the primaries admitting a primary descendant, the one with $\Delta^{\prime}=\frac{d}{2}-1$ and $\ell^{\prime}=0$ has the same quantum numbers of a scalar free field $\phi_{f}$ in $d$ space-time dimensions. Is there any restriction in a unitary theory assuming this to be a free field? The answer is no, of course, but since this fact is not always clearly stated in literature, we quote here a theorem due to Weinberg [58]: in a scale-invariant relativistic theory any local scalar operator $\psi(x)$ having scaling dimension $\Delta_{\psi}=\frac{d}{2}-1$ is necessarily a free field:

$$
\partial^{2} \psi(x)=0
$$

\footnotetext{
${ }^{3}$ As already mentioned, such a list applies to primaries that belong to the traceless symmetric representations of $\mathrm{SO}(d)$. For more general representations higher order Casimir invariants are necessary.
} 


\begin{tabular}{|c|c|c|c|}
\hline Parent primary & \multicolumn{2}{|c|}{ Primary descendant } & \\
\hline$\Delta_{k}^{\prime}$ & $\Delta_{k}$ & $\ell$ & \\
\hline $1-\ell^{\prime}-k$ & $1-\ell+k$ & $\ell^{\prime}+k$ & $k=1,2, \ldots$ \\
$\frac{d}{2}-k$ & $\frac{d}{2}+k$ & $\ell^{\prime}$ & $k=1,2, \ldots$ \\
$d+\ell^{\prime}-k-1$ & $d+\ell+k-1$ & $\ell^{\prime}-k$ & $k=1,2, \ldots, \ell$ \\
\hline
\end{tabular}

Table 1. List of solutions of the algebraic system (2.2). The $\Delta_{k}^{\prime}$ 's in the first column give the positions of poles of a generic conformal block defined in (2.1). The second and third columns list the scaling dimension $\Delta_{k}$ and the spin $\ell$ of the primary descendant belonging to the representation generated by the parent primary $\left|\Delta^{\prime}, \ell^{\prime}\right\rangle$.

It is interesting to note that in this way the equation of motion of a free field has been obtained in a purely group-theoretical manner, with no reference to Lagrangians or to any sort of dynamical principles.

Ref. [57] obtained the explicit form of the polynomials in the Lie algebra elements which generate null states when applied to the primaries listed in the first column of table 1. That transforming the primary state $\left|\frac{d}{2}-1,0\right\rangle$ into the corresponding null state of the second column of the table is $P^{2}\left|\frac{d}{2}-1,0\right\rangle \equiv-\partial^{2}\left|\frac{d}{2}-1,0\right\rangle=\left|\frac{d}{2}+1,0\right\rangle$ which in view of the above theorem is actually zero. Therefore the conformal multiplet generated by a bosonic free field $\phi_{f}$ is short. Turning on the interaction by letting $\phi$ acquire anomalous dimensions implies that this descendant is no longer zero. However, strictly speaking, it is no longer a null state; in fact its norm is [57]

$$
\left\langle\Delta, 0\left|K^{2} P^{2}\right| \Delta, 0\right\rangle=8 d \Delta\left(\Delta-\Delta_{\phi_{f}}\right)
$$

as a consequence of the commutation relations

$$
\left[K_{\mu}, P_{\nu}\right]=2 i\left(\eta_{\mu \nu} D-J_{\mu \nu}\right)
$$

combined with the constraints

$$
K_{\mu}|\Delta, 0\rangle=J_{\mu \nu}|\Delta, 0\rangle=0, \quad\langle\Delta, 0| P_{\mu}=\langle\Delta, 0| J_{\mu \nu}=0, \quad\langle\Delta, 0 \mid \Delta, 0\rangle=1 .
$$

Putting $\Delta=\Delta_{\phi}=\Delta_{\phi_{f}}+\gamma_{\phi}$, we see that this state becomes null when it approaches the free field theory, where it disappears. This fact has an important role in the study of the possible smooth deformations of a free theory. As mentioned in the introduction, we say that a free field theory in $d$ dimensions admits a smooth deformation in $d-\epsilon$ if there is a one-to-one mapping to another CFT in which any local operator $\mathcal{O}_{f}$ of the free theory corresponds to an operator $\mathcal{O}$ of the deformed theory with the same spin and $\lim _{\epsilon \rightarrow 0} \Delta_{\mathcal{O}}=\Delta_{\mathcal{O}_{f}}$. For a general $d$ such a deformation does not exists because $\partial^{2} \phi$ does not have a corresponding operator in the free theory. There are however special values of $d$, where there is a scalar $\phi_{f}^{m-1}$ having the same scaling dimensions of the vanishing operator $\partial^{2} \phi_{f}$ which restores the one-to-one correspondence. Thus from the degeneracy condition $(m-1) \Delta_{\phi_{f}}=\Delta_{\phi_{f}}+2$ the upper critical dimension (1.10) follows. 


\section{Spinning operators}

In this section we use the methods and the notations of [57] to derive some useful properties of local operators belonging to symmetric and traceless representations of $\mathrm{SO}(d)$. A primary operator of $\operatorname{spin} \ell$ is denoted by

$$
\mathcal{O}(x, z)=\mathcal{O}^{\mu_{1} \ldots \mu_{\ell}}(x) z_{\mu_{1}} \ldots z_{\mu_{\ell}}
$$

where $z_{\mu}$ is a vector of $\mathbb{C}^{d}$ that can be consistently assumed to satisfy $z \cdot z=0$ as a consequence of the null traces of the tensor (3.1). One can recover the tensor indices by applying recursively the Todorov differential operator defined as [60]

$$
D_{z}^{\mu} \equiv\left(\nu+z \cdot \frac{\partial}{\partial z}\right) \frac{\partial}{\partial z_{\mu}}-\frac{1}{2} z^{\mu} \frac{\partial^{2}}{\partial z \cdot \partial z} .
$$

where $\nu=\frac{d}{2}-1$.

The two-point function of a canonically normalized primary of spin $\ell$ and scaling dimensions $\Delta$ is

$$
\left\langle\mathcal{O}\left(x_{1}, z_{1}\right) \mathcal{O}\left(x_{2}, z_{2}\right)\right\rangle=\frac{\left(z_{1}^{\mu} I_{\mu \nu}\left(x_{12}\right) z_{2}^{\nu}\right)^{\ell}}{\left(x_{12}^{2}\right)^{\Delta}}
$$

with $x_{i j} \equiv x_{i}-x_{j}$ and $I_{\mu \nu}(x) \equiv \delta_{\mu \nu}-2 x_{\mu} x_{\nu} / x^{2}$.

The three-point of the spinning primary $\mathcal{O}_{1}\left(x_{1}, z\right)$ with two scalar primaries $\mathcal{O}_{2}\left(x_{2}\right)$ and $\mathcal{O}_{3}\left(x_{3}\right)$ reads

$$
\left\langle\mathcal{O}_{1}\left(x_{1}, z\right) \mathcal{O}_{2}\left(x_{2}\right) \mathcal{O}_{3}\left(x_{3}\right)\right\rangle=\frac{\mathrm{c}_{123}(z \cdot y)^{\ell}}{\left(x_{12}^{2}\right)^{\frac{\Delta_{1}+\Delta_{2}-\Delta_{3}-\ell}{2}}\left(x_{23}^{2}\right)^{\frac{\Delta_{2}+\Delta_{3}-\Delta_{1}+\ell}{2}}\left(x_{31}^{2}\right)^{\frac{\Delta_{3}+\Delta_{1}-\Delta_{2}-\ell}{2}}},
$$

with $y^{\mu} \equiv \frac{x_{31}^{\mu}}{x_{31}^{2}}-\frac{x_{12}^{\mu}}{x_{12}^{2}}$. We can infer the OPE

$$
\mathcal{O}_{1}(x, z) \mathcal{O}_{2}(0)=\mathrm{c}_{123} \frac{(-x \cdot z)^{\ell}}{\left(x^{2}\right)^{\frac{\Delta_{1}+\Delta_{2}-\Delta_{3}+\ell}{2}}} \mathcal{O}_{3}(0)+\ldots,
$$

indeed inserting this OPE in the 1.h.s. of (3.4) with $x^{\mu}=x_{1}^{\mu}, x_{2}^{\mu}=0$ and $x^{2} \ll x_{3}^{2}$ yields the r.h.s. .

We want to exploit this OPE to obtain two important and useful properties of the conserved spinning operators (see also [59]). Within these notations the conservation law of $\mathcal{J}_{\ell}(x, z) \equiv \mathcal{O}_{1}(x, z)$ reads

$$
\frac{\partial}{\partial x} \cdot D_{z} \mathcal{J}_{\ell}(x, z)=0
$$

Applying this differential operator to both sides of (3.5) gives

$$
\mathrm{c}_{\mathcal{J O}_{2} \mathcal{O}_{3}}(d-4+2 \ell)\left(\Delta_{\ell}+\Delta_{2}-\Delta_{3}-d+2-\ell\right)=0 .
$$

For $\Delta_{2}=\Delta_{3}$ we obtain $\Delta_{\ell}=d-2+\ell$, the well known scaling dimension of a conserved HS current. For $\Delta_{2} \neq \Delta_{3}$ the above equation tells us that the OPE coefficient must vanish:

$$
\frac{\partial}{\partial x} \cdot D_{z} \mathcal{J}_{\ell}=0 \& \Delta_{2} \neq \Delta_{3} \Rightarrow \mathrm{c}_{\mathcal{J O}_{2} \mathcal{O}_{3}}=0
$$


Such a theorem will be useful in our derivation of the anomalous dimensions of weakly broken HS currents.

We write now the previous OPE in the form

$$
\phi^{p}(0) \phi(x)=c_{p \ell} \frac{(x \cdot z)^{\ell}}{\left(x^{2}\right)^{\frac{\Delta_{\phi} p+\Delta_{\phi}-\Delta_{p, \ell}+\ell}{2}}} \mathcal{O}_{p, \ell}(0, z)+\ldots,
$$

where $\mathcal{O}_{p, \ell}$ is a spinning primary with scaling dimensions $\Delta_{p, \ell}=(p+1) \Delta_{\phi_{f}}+\gamma_{p, \ell}$ in $d-\epsilon$ as in (1.2), $\phi$ is an interacting scalar field and $\phi^{p}$ is normalized in such a way that

$$
\left\langle\phi^{p}(x) \phi^{p}(0)\right\rangle=\frac{1}{\left(x^{2}\right)^{\Delta_{\phi^{p}}}} .
$$

A simple example of a spinning primary of the free field theory is

$$
\mathcal{O}_{2,2}^{f}=\mathcal{N}\left[\phi_{f}\left(z \cdot \partial \phi_{f}\right)^{2}-\frac{d-2}{d} \phi_{f}^{2}(z \cdot \partial)^{2} \phi_{f}\right],
$$

where $\mathcal{N}$ is a normalization factor. Notice that there is an overall sign ambiguity in the definition of $\mathcal{O}_{p, \ell}$ which entails a sign ambiguity in the OPE coefficients. The anomalous dimension of $\mathcal{O}_{p, \ell}$ depends on ratios of these c's, so this sign ambiguity as well as the dependence on the normalization factor $\mathcal{N}$ disappear.

The computed anomalous dimensions of this kind of spinning operators are explicitly written in eq.s (4.8) and (4.9) . It is worth to stress that such a result is only valid for a primary operator. For instance $\left[\phi^{3}\right]$ has a descendant of spin 2 which is degenerate with the above primary in the Gaussian limit, while its anomalous dimension is $\gamma_{\phi^{3}}$ (see eq. (4.5)), which is very different from (4.8) and (4.9). ${ }^{4}$

Using the explicit expression (3.11) it is easy to evaluate $c_{2,2}$, however this direct method would imply lengthy calculations for general $c_{p, \ell}$. A much simpler approach is based on the following observation: all the $\mathcal{O}_{p, \ell}$ we are interested in are generated in the free field theory by the fusion rule

$$
\left[\phi_{f}^{p}\right] \times\left[\phi_{f}\right]=\sqrt{p}\left[\phi_{f}^{p-1}\right]+\sum_{\ell=0}^{\infty} \mathrm{c}_{p, \ell}\left[\mathcal{O}_{p, \ell}^{f}\right] .
$$

where the spinning operators $\mathcal{O}_{p, \ell}^{f}$ are built with $\ell$ derivatives acting on $p+1$ copies of $\phi_{f}$, hence their dimension is $(p+1) \frac{d-2}{2}+\ell$. Contrarily to what happens in the more general fusion rule $\left[\phi_{f}^{p}\right] \times\left[\phi_{f}^{q}\right]$ with $q>1$, it turns out that the indices of derivatives are not contracted each other, then the spin of these operators coincides with the number of derivatives. This dramatically simplifies the computation of the $c_{p, \ell}$ 's. Actually appendix A describes a way to obtain these coefficients. The result is

$$
c_{p, \ell}^{2}=\frac{p(\nu)_{\ell}(2 \nu)_{\ell}+(-1)^{\ell}(2 \nu)_{\ell}(p \nu)_{\ell}}{\ell !((p+1) \nu+\ell-1)_{\ell}}
$$

\footnotetext{
${ }^{4}$ Notice that the spectrum of local operators of the free-field theory is highly degenerate at large $\ell$. This degeneracy is completely removed at the WF fixed point.
} 
where $(x)_{y} \equiv \frac{\Gamma(x+y)}{\Gamma(x)}$ is the Pochhammer symbol and $\nu=\frac{d}{2}-1$. In the interacting theory we have

$$
\left\langle\phi^{p}\left|\mathcal{O}_{p, \ell}\right| \phi\right\rangle=\mathrm{c}_{p, \ell}+O(\epsilon) .
$$

It is important to notice that in the free field theory, for large values of $p$ and $\ell$, the spectrum of this spinning operators is highly degenerate: there are many inequivalent ways to build a primary. There are however two infinite families of non-degenerate operators, namely $\ell<4$ and $p$ arbitrary, or $p<3$ and $\ell$ arbitrary, as shown in [61]. We shall see in the next section how to explicitly calculate the anomalous dimensions of all the primaries belonging to these two families.

In accordance with (1.7) we need to compute $\left\langle\phi^{p}\left|\mathcal{O}_{p, \ell}\right| \chi\right\rangle \equiv\left\langle\phi^{p}\left|\mathcal{O}_{p, \ell} P^{2}\right| \phi\right\rangle$, where $\left\langle\phi^{p}\right|=\lim _{x^{2} \rightarrow \infty}\langle 0| \phi^{p}(x)\left(x^{2}\right)^{\Delta_{\phi^{p}}}$. Here $|\chi\rangle=\lim _{x \rightarrow 0} P^{2} \phi(x)|0\rangle$ and $\mathcal{O}_{p, \ell}(x, z)$ is evaluated at $x^{2}=1$ and $z \cdot x=1$, so that the associated three-point function coincides numerically with the OPE coefficient. Operating with $P^{2}=-\partial^{2}$ on both sides of (3.9) it follows at once

$$
\left\langle\phi^{p}\left|\mathcal{O}_{p, \ell}\right| \chi\right\rangle=\mathrm{M}_{p, \ell}\left\langle\phi^{p}\left|\mathcal{O}_{p, \ell}\right| \phi\right\rangle
$$

with $^{5}$

$$
\begin{aligned}
\mathrm{M}_{p, \ell} & =\left(\Delta_{\phi}+\Delta_{\phi^{p}}-\Delta_{p, \ell}+\ell\right)\left(\Delta_{p, \ell}-\Delta_{\phi}-\Delta_{\phi^{p}}-2+d+\ell\right) \\
& =\left(\gamma_{\phi}+\gamma_{\phi^{p}}-\gamma_{p, \ell}\right)(d-2+2 \ell)+O\left(\epsilon^{2}\right) .
\end{aligned}
$$

In our study of certain five-point functions we also need to know $\left\langle\chi\left|\mathcal{J}_{\ell}\right| \chi\right\rangle \equiv\left\langle\phi\left|K^{2} \mathcal{J}_{\ell} P^{2}\right| \phi\right\rangle$. Repeating the above calculation we find

$$
\left\langle\chi\left|\mathcal{J}_{\ell}\right| \chi\right\rangle=\mathrm{N}_{\ell}\left\langle\phi\left|\mathcal{J}_{\ell}\right| \phi\right\rangle
$$

with

$$
\begin{aligned}
\mathrm{N}_{\ell} & =\mathrm{M}_{1, \ell}\left(2 \Delta_{\phi}-\Delta_{1, \ell}+2+\ell\right)\left(\Delta_{1, \ell}-2 \Delta_{\phi}+d-4+\ell\right) \\
& =2\left(2 \gamma_{\phi}-\gamma_{\ell}\right)(d-2+2 \ell)\left(\gamma_{\ell}-2 \gamma_{\phi}+d-4+2 \ell\right)+O\left(\epsilon^{3}\right) .
\end{aligned}
$$

The denominator of (1.7) has been already computed in (2.5); it reads

$$
\frac{1}{\langle\chi \mid \chi\rangle} \equiv \frac{1}{\left\langle\phi\left|K^{2} P^{2}\right| \phi\right\rangle}=\frac{\mathrm{Q}}{\Delta_{\phi}-\Delta_{\phi_{f}}}=\frac{\mathrm{Q}}{\gamma_{\phi}}
$$

with

$$
\mathrm{Q}=\frac{1}{4 d(d-2)}+O(\epsilon)
$$

Equations (3.16), (3.18) and (3.20) are the main ingredients to formulate the matching conditions discussed in the next section.

\footnotetext{
${ }^{5}$ Notice that $\mathrm{M}$ is very similar, but not identical to the analogous quantity calculated in [57]. There it referred to a primary descendant of a spinning operator that appears in the OPE of two scalars. Here the spinning primary is an external operator and M refers to $\chi$, the primary descendant of $\phi$.
} 


\section{Matching conditions}

The free field theory of a single real scalar is invariant under the $\mathbb{Z}_{2}$ transformation $\phi_{f} \rightarrow$ $-\phi_{f}$, therefore the fusion rule (1.3) can be only generalized to those upper dimensions $d_{m}$ where the primary descendant $\phi_{f}^{m-1}$ is an odd power $2 q+1=m-1$ of $\phi$

$$
\left[\mathcal{O}_{p, \ell}^{f}\right] \times\left[\phi_{f}^{p}\right]=\mathrm{c}_{\mathcal{O}_{p, \ell} \phi^{p} \phi}\left[\phi_{f}\right]+\mathrm{c}_{\mathcal{O}_{p, \ell} \phi^{p} \phi^{2 q+1}}\left[\phi_{f}^{2 q+1}\right]+\ldots
$$

The first OPE coefficients $\boldsymbol{c}_{\mathcal{O}_{p, \ell} \phi^{p} \phi} \equiv c_{p, l}$ can be extracted from the fusion $\left[\phi_{f}^{p}\right] \times\left[\phi_{f}\right]$ as we saw in the previous section (see eq. (3.13)). Similarly the second OPE coefficients $\mathrm{c}_{\mathcal{O}_{p, \ell} \phi^{p} \phi^{2 q+1}} \equiv \mathrm{c}_{q, p, l}$ can be computed from the fusion $\left[\phi_{f}^{p}\right] \times\left[\phi_{f}^{2 q+1}\right]$. More specifically, as eq. (A.4) shows, they are the coefficients of the following conformally invariant function

$$
\begin{array}{r}
\frac{p !(2 q+1) !}{(q !)^{2}} u^{\nu(p+1) / 2} \sum_{k=0}^{q+1} \frac{v^{-k \nu}}{(k !)^{2}(p-q-k) !(q-k+1) !} \\
=\sum_{n=0}^{\infty} \sum_{\ell=0}^{\infty} \mathrm{c}_{n, q, p, \ell}^{2} G_{\nu(p+1)+2 n+\ell, \ell}(u, v),
\end{array}
$$

where the $G_{\Delta, \ell}$ 's are the conformal blocks contributing to the $\phi_{f}^{p} \phi_{f}^{2 q+1}$ channel of the fourpoint function $\left\langle\phi_{f}^{p} \phi_{f}^{2 q+1} \phi_{f}^{p} \phi_{f}^{2 q+1}\right\rangle$ and $\mathrm{c}_{0, q, p, \ell} \equiv \mathrm{c}_{q, p, \ell}$. Unfortunately we did not find a closed expression for these coefficients for general $q$ and $\ell$.

The matching conditions (1.8) can be written more precisely in the form

$$
\lim _{\epsilon \rightarrow 0} \frac{\mathrm{M}_{p, \ell}^{2} \mathrm{Q}}{\gamma_{\phi}}=\left(\frac{\mathrm{c}_{q, p, \ell}}{\mathrm{c}_{p, \ell}}\right)^{2},
$$

where $\mathrm{M}_{p, \ell}$ and $\mathrm{Q}$ are defined in (3.16) and (3.20).

When $\ell=0$, then $\gamma_{p, 0} \equiv \gamma_{\phi^{p+1}}$ and eq.s (3.13) and (4.2) give at once

$$
\frac{\mathrm{c}_{q, p, 0}}{\mathrm{c}_{p, 0}}=\left(\begin{array}{l}
p \\
q
\end{array}\right) \frac{\sqrt{(2 q+1) !}}{(q+1) !} .
$$

In such a case the matching conditions define a set of recursion relations that have been completely solved in [45]. The solution reads

$$
\gamma_{\phi^{p}}^{(1)}=\frac{q(p-q)_{q+1}}{(q+1)_{q+1}}, \gamma_{\phi}^{(2)}=2 q^{2}\left[\frac{((q+1) !)^{2}}{(2(q+1)) !}\right]^{3} .
$$

Thus, the only remaining unknown in (4.3) is $\gamma_{p, \ell}$. This equation can be further simplified considering the four-point function $\left\langle\phi^{q} \phi^{q+1} \mathcal{O}_{p, \ell} \phi^{p}\right\rangle$. The associated matching condition reads

$$
\lim _{\epsilon \rightarrow 0} \frac{\mathrm{M}_{q, 0} \mathrm{M}_{p, \ell} Q}{\gamma_{\phi}}=\frac{\mathrm{c}_{q, p, \ell}}{\mathrm{c}_{p, \ell}} \frac{\mathrm{c}_{q, q, 0}}{\mathrm{c}_{q, 0}} .
$$

Now this equation is linear in $\gamma_{p, \ell}$ and the solution is

$$
\gamma_{p, \ell}^{(1)}=\gamma_{\phi^{p}}^{(1)}+4 \gamma_{\phi}^{(2)} \frac{\mathrm{c}_{q, p, \ell}}{\mathrm{c}_{p, \ell}} \frac{1+q}{q(1+q \ell)} \frac{(\sqrt{(2 q+1) !})^{3}}{((q+1) !)^{2}} ;
$$


$\mathrm{c}_{p, \ell}$ is given in (3.13) and $\mathrm{c}_{q, p, \ell}$ is implicitly defined in (4.2). Actually, when $\mathcal{O}_{p, \ell}$ is degenerate this equation is of no practical use. In such a case the fusion rule (3.12) we used to construct $\mathcal{O}_{p, \ell}$ does not select a single primary, but a linear combination of them; these have different anomalous dimensions in $d-\epsilon$, therefore when both $p$ and $\ell$ are large it does not apply. ${ }^{6}$ Let us see some specific examples where it works. There is no primary of spin $\ell=1$ in a theory with a single real scalar. For $d=4-\epsilon$ we have for any $p$ and any $\ell>1$

$$
\gamma_{p, 2}^{(1)}=\frac{(p-1)(4+3 p)}{18}, \gamma_{p, 3}^{(1)}=\frac{\left(p^{2}-3\right)}{6}, \gamma_{2, \ell}^{(1)}=\frac{1}{3}+\frac{2(-1)^{\ell}}{3(\ell+1)} .
$$

Similarly for $d=3-\epsilon$

$$
\gamma_{p, 2}^{(1)}=\frac{(p-1)(p-2)(5 p+18)}{150}, \gamma_{p, 3}^{(1)}=\frac{(p-2)\left(7 p^{2}+11 p-90\right)}{210}, \gamma_{2, \ell}^{(1)}=0 .
$$

In $d=4-\epsilon$ and $p<12$ these $\gamma$ 's have been also computed in [61] and the results agree. When $\ell>3$ and $p>2$ these operators are degenerate in the free theory limit. For instance for $\ell=4$ and $p>2$ the space of primaries has dimension two [61], while the equation above reads

$$
\gamma_{p, 4}^{(1)}=\frac{p(p-1)}{6}+\frac{\sqrt{2(p-1)\left(2 p^{3}-11 p^{2}+78 p-104\right)}}{15 \sqrt{p^{2}+p+6}} .
$$

It gives the exact value for $p=2$ and an intermediate value between the two exact $\gamma$ 's in the other cases. For large values of $\ell$ one could apply the inversion formula of [62] (see also [63]) to extract the OPE coefficients of (4.2) in this limit.

In accordance with the theorem (3.8) we have, in the case $p=1$,

$$
\mathrm{c}_{q, 1, \ell} \equiv \mathrm{c}_{\mathcal{J}_{\ell} \phi \phi^{2 q+1}}=0,
$$

moreover $\gamma_{\phi}^{(1)}=0$, then $\gamma_{p, \ell}^{(1)}=0 .^{7}$

Further information on the anomalous dimensions of $\mathcal{J}_{\ell}$ can be gained by studying the five point function $\left\langle\phi^{q} \phi^{q+1} \mathcal{J}_{\ell} \phi^{q} \phi^{q+1}\right\rangle$. In the factorization scheme shown in figure 1 we have

$$
\left\langle\phi^{q} \phi^{q+1} \mathcal{J}_{\ell} \phi^{q} \phi^{q+1}\right\rangle=\sum_{\mathcal{O}} \sum_{\mathcal{O}^{\prime}} \operatorname{c}_{\mathcal{O}} c_{\mathcal{O}^{\prime}} c_{\mathcal{J}} \sum_{\alpha \in \mathcal{H}_{\mathcal{O}}} \sum_{\beta \in \mathcal{H}_{\mathcal{O}^{\prime}}} \frac{\left\langle\phi^{q} \phi^{q+1} \mid \alpha\right\rangle\left\langle\alpha\left|\mathcal{J}_{\ell}\right| \beta\right\rangle\left\langle\beta \mid \phi^{q} \phi^{q+1}\right\rangle}{\langle\alpha \mid \alpha\rangle\langle\beta \mid \beta\rangle},
$$

where $\boldsymbol{c}_{\mathcal{O}} \equiv \mathrm{c}_{\mathcal{O}^{q} \phi^{q+1}}$ and $\mathrm{c}_{\mathcal{J}} \equiv \mathrm{c}_{\mathcal{O O}^{\prime} \mathcal{J}_{\ell}}$. In view of the already mentioned theorem (3.8), if $\mathcal{J}_{\ell}$ is conserved and $\mathcal{O}, \mathcal{O}^{\prime}$ are scalars, then $\mathrm{c}_{\mathcal{O O}^{\prime} \mathcal{J}_{\ell}} \propto \delta_{\mathcal{O}, \mathcal{O}^{\prime}}$. In particular, in the free field theory the two factorization channels always contain the scalars $\phi_{f}$ and $\phi_{f}^{2 q+1}$. The OPE coefficients of these two contributions are respectively $\mathrm{c}_{\phi_{f} \phi_{f}^{q} \phi_{f}^{q+1}}^{2} \mathrm{c}_{\phi_{f} \phi_{f}} \mathcal{J}_{\ell} \equiv \mathrm{c}_{q, 0}^{2} \mathrm{c}_{\phi_{f} \phi_{f} \mathcal{J}_{\ell}}$ and

\footnotetext{
${ }^{6}$ I thank L.F. Alday for this important remark and for pointing out ref. [61] to me. Note that eq. (4.7) is still true even in the degenerate case, provided we assume that $\mathcal{O}_{p, \ell}$ is an eigenfunction of $D$ in $d-\epsilon$ and the OPE coefficients $c_{p, \ell}$ and $c_{q, p, \ell}$ are computed with this operator.

${ }^{7}$ This is only true for the upper critical dimensions $d=2(q+1) / q$. For the more general case $d=$ $2 m /(m-2)$, which includes $d=6$, the above matching conditions do not apply. Nevertheless we shall extract the anomalous dimensions of $\mathcal{J}_{\ell}$ also in such a case.
} 


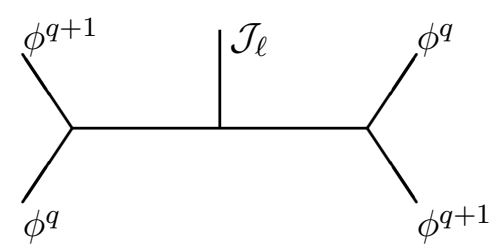

Figure 1. The factorization channels of the five-point under study.

$\mathrm{c}_{\phi_{f}^{2 q+1}}^{2} \phi_{f}^{q} \phi_{f}^{q+1} \mathrm{c}_{\phi_{f}^{2 q+1}} \phi_{f}^{2 q+1} \mathcal{J}_{\ell}=(2 q+1) \mathrm{c}_{q, q, 0}^{2} \mathrm{c}_{\phi_{f} \phi_{f}} \mathcal{J}_{\ell}$, where we used the useful identity (see appendix)

$$
\frac{\mathrm{c}_{\phi_{f}^{p}} \phi_{f}^{p} \mathcal{J}_{\ell}}{\mathrm{c}_{\phi_{f} \phi_{f} \mathcal{J}_{\ell}}}=p
$$

Turning on the interaction, $\phi^{2 q+1}$ becomes a descendant of $\phi$ and the second contribution has to be absorbed by the first, in accordance with (1.7). Therefore the matching condition of the five-point function under study takes the form

$$
\lim _{\epsilon \rightarrow 0} \frac{M_{q, 0}^{2} \mathrm{~N}_{\ell} \mathrm{Q}^{2}}{\gamma_{\phi}^{2}}=(2 q+1)\left(\frac{\mathrm{c}_{q, q, 0}}{\mathrm{c}_{q, 0}}\right)^{2} .
$$

When combined with (4.3) it leads to

$$
\lim _{\epsilon \rightarrow 0} \frac{\mathrm{N}_{\ell} \mathrm{Q}}{\gamma_{\phi}}=2 q+1
$$

On substituting (3.18) we find at once

$$
\gamma_{\ell}^{(2)}=2 \gamma_{\phi}^{(2)}\left(1-\frac{(\nu+1)(\nu+2)}{(\ell+\nu)(\ell+\nu-1)}\right)
$$

with $\nu=\frac{d}{2}-1=\frac{1}{q}$. The above reproduces the correct second-order anomalous dimension of $\mathcal{J}_{\ell}$ at $d=4-\epsilon$ [37]. This expression is true more generally at the multicritical WF fixed points at $d=2(q+1) / q-\epsilon$ which include the physically relevant case at $d=3-\epsilon$. In all the cases we have $\gamma_{2}^{(2)}=0$, as expected by the conservation of the stress tensor at the WF fixed point.

There is a simple modification that allows to generalize the above matching condition to the general case of the upper critical dimension $d_{m}=2 m /(m-2)$. Let us see for simplicity the case $d=6-\epsilon$, where the scalar becoming a descendant of $\phi$ is $\phi^{2}$. At the Gaussian fixed point we have the fusion rule $\left[\phi_{f}\right] \times\left[\phi_{f}\right]=1+\sqrt{2}\left[\phi_{f}^{2}\right]+$ spinning primaries. In the interacting theory $\phi^{2}$ is no longer a primary, hence it should be replaced by $\phi$, but $\lim _{\epsilon \rightarrow 0} \mathrm{c}_{\phi \phi \phi}=0$. The resulting matching condition of the four-point $\langle\phi \phi \phi \phi\rangle$ reads $^{8}$

$$
\lim _{\epsilon \rightarrow 0} \mathrm{c}_{\phi \phi \phi}^{2} \frac{\mathrm{Q} \Delta_{\phi}\left(\Delta_{\phi}+2-d\right)}{\gamma_{\phi}}=2
$$

It can be used to express the OPE coefficient $c_{\phi \phi \phi}$ of the interacting theory in terms of the anomalous dimensions of $\phi$. More generally, at a WF fixed point in which the primary

\footnotetext{
${ }^{8}$ Such a relation has been first pointed out by Yu Nakayama in some unpublished notes. I thank him for sharing with me his notes.
} 
descendant $\phi^{m-1}$ is a even power $m-1=2 r$ we have

$$
\lim _{\epsilon \rightarrow 0} c_{\phi^{r} \phi^{r} \phi}^{2} \frac{\mathrm{Q} \Delta_{\phi}\left(\Delta_{\phi}+2-d\right)}{\gamma_{\phi}}=\frac{(2 r) !}{(r !)^{2}},
$$

with $d=\frac{2(2 r+1)}{2 r-1}-\epsilon$. The matching condition of the five-point function $\left\langle\phi^{r} \phi^{r} \mathcal{J}_{\ell} \phi^{r} \phi^{r}\right\rangle$, when combined with (4.18) and (4.13) gives the promised generalization

$$
\lim _{\epsilon \rightarrow 0} \frac{\mathrm{N}_{\ell} \mathrm{Q}}{\gamma_{\phi}}=m-1=\frac{d_{m}+2}{d_{m}-2},
$$

valid for any integer $m$. Notice however that the first non-vanishing anomalous dimensions of $\mathcal{J}_{\ell}$ in $d=6-\epsilon$ are $O(\epsilon)$, since in this case, unlike what happens at $d=4-\epsilon$, at $d=3-\epsilon$ and at the other multicritical fixed points described by $(4.5)$, now $\gamma^{(1)} \neq 0$. Precisely we have $\gamma_{\phi}^{(1)}=-\frac{1}{18}$, so the equation above yields

$$
\gamma_{\ell}^{(1)}=-\frac{1}{9}\left(1-\frac{12}{(\ell+1)(\ell+2)}\right),
$$

which exactly agrees with the known results [53]. The minus sign reflects the fact that this WF fixed point is described by a non-unitary CFT.

The matching condition (4.19) applies also when $\ell=0$, where it gives a new equation ${ }^{9}$ for the anomalous dimension of $\mathcal{J}_{0} \equiv \phi^{2}$. This is useful in the multicritical case, where $\gamma_{\phi^{2}}^{(1)}$ is vanishing (see eq. (4.5)). This new equation gives

$$
\gamma_{\phi^{2}}^{(2)}=\frac{4 q(2+q)}{(q-1)} \gamma_{\phi}^{(2)}=\frac{8 q^{3}(q+2)}{q-1}\left[\frac{((q+1) !)^{2}}{(2(q+1)) !}\right]^{3} .
$$

In particular the $d=3$ scalar theory perturbed with a $\phi^{6}$ potential (i.e. $q=2$ ) gives $\gamma_{\phi^{2}}^{(2)}=\frac{4}{125}$, as can be directly checked by putting $\ell=0$ in (4.16). Eq. (4.21) agrees with the results obtained with more conventional methods based on the Lagrangian formulation and equations of motion [46].

\section{Conclusions}

In the present paper we studied some physical properties of perturbative Wilson-Fisher fixed points under the only assumption of their conformal invariance. Using a generalization of the method developed in $[43,45]$, we computed, to the first non-trivial order in the $\epsilon$ expansion, the anomalous dimensions of a large set spinning operators $\mathcal{O}_{p, \ell}$ that in the Gaussian theory have scaling dimensions $\Delta=(p+1)\left(\frac{d}{2}-1\right)+\ell$. In accordance with the bootstrap philosophy no Lagrangian was assumed. The interaction was implicitly turned on by assuming that the local operators acquire anomalous dimensions. The WF fixed points were defined using only conformal invariant concepts, with no reference to the renormalization group. They were seen as smooth conformal deformations of scalar freefield theories (see the precise definition around eq. (1.1)), where it was somewhat hidden the notion of UV and IR fixed points of the RG.

\footnotetext{
${ }^{9}$ The only exception is $d=6-\epsilon$ because $\phi^{2}$ is not a primary there.
} 
All the computations of the present paper rely on a unique group-theoretical mechanism, the conformal multiplet recombination. Schematically, it tells us that the free field scalar $\phi_{f}$ corresponds to a short multiplet, while the multiplet of the corresponding interacting field is long; in the $\epsilon \rightarrow 0$ limit thy must agree. We derived in this way two sets of matching conditions - (4.3) and (4.19) — which encode the wanted information on $\mathcal{O}_{\Delta, \ell}$. The former describes the matching conditions of certain four-point functions which generalize to $\ell \neq 0$ those already considered in $[43,45]$. The latter is completely new and is obtained by matching conditions of the five-point function sketched in figure 1 . We used it to compute the anomalous dimensions of the subclass of spinning operators $\mathcal{J}_{\ell} \equiv \mathcal{O}_{1, \ell}$; they are conserved an the Gaussian fixed point, while acquire anomalous dimensions at the WF fixed point. The resulting single equation (4.19) tells us that the stress tensor is automatically conserved at any (permitted) space-time dimension, while for $\ell \neq 2$ yields the first non-vanishing contribution of the anomalous dimensions of the HS currents. In the more conventional approaches these results require different Lagrangians with different perturbing potentials, namely $\phi^{4}$ in $d=4-\epsilon, \phi^{6}$ in $d=3-\epsilon$, and $\phi^{3}$ in $d=6-\epsilon$.

There are several directions one can pursue. The most obvious one is to extend our analysis to $O(N)$ invariant models. Another interesting extension would be to apply this approach to generalized free-field theories, following the line initiated in $[43,45]$ for the scalar primaries. Notice that in the present approach, unlike other conformal bootstrap calculations, the crossing symmetry has not been used. Enforcing this powerful symmetry in our scheme could hopefully generate new results.

It would be finally important to find some connection of our method to other bootstrapinspired analytic approaches. In an elegant study which relies on turning the crossing equations into an algebraic problem [55, 56], the HS symmetry is explicitly broken by introducing a small parameter $g$. At first non-trivial order in $g$ the spectrum of weakly broken currents follows. Unfortunately this method cannot apply to more general, non conserved, spinning operators; notice also that the conservation of the stress tensor does not follow automatically, and has to be assumed as a further constraint. It would be interesting to combine such an algebraic form of the crossing equations with the mechanism of conformal multiplet recombination used here. Another powerful way to obtain analytic information about the spectrum of the primary operators in a WF fixed point relies upon a Mellin space representation of correlation functions [48-50]. It gives at the moment the analytic results of higher order in the anomalous dimensions and OPE coefficients. So far it has been only applied to the four-point function $\langle\phi \phi \phi \phi\rangle$. An extension of the analysis to $\left\langle\phi^{p} \phi \phi^{p} \phi\right\rangle$ would provide information on a much larger class of spinning operators.

\section{Acknowledgments}

The author is grateful to A. L. Guerrieri, A. C. Petkou and C. Wen for fruitful discussions and comments on the manuscript. He would also like to thank L. F. Alday, S. Hikami and M. Meineri for useful discussions and correspondence. 


\section{A OPE coefficients of the free-field theory}

In this appendix we collect some useful formulae on the conformal block expansions and OPE coefficients of a scalar free field theory. Here $\phi$ is the free fundamental scalar and $\phi^{p}$ denotes the normal ordered scalar primary $\frac{:(\phi)^{p}:}{\sqrt{p !}}$. The normalization is chosen in such a way that

$$
\left\langle\phi^{p}\left(x_{1}\right) \phi^{p}\left(x_{2}\right)\right\rangle=\frac{1}{\left(x_{12}^{2}\right)^{p \nu}} .
$$

Applying the Wick's contractions to the four-point function $\left\langle\phi^{p}\left(x_{1}\right) \phi^{r}\left(x_{2}\right) \phi^{p}\left(x_{3}\right) \phi^{r}\left(x_{4}\right)\right\rangle$ with $p \geq r$ and comparing it with the standard parameterization of a generic CFT, namely,

$$
\left\langle\mathcal{O}_{1}\left(x_{1}\right) \mathcal{O}_{2}\left(x_{2}\right) \mathcal{O}_{3}\left(x_{3}\right) \mathcal{O}_{4}\left(x_{4}\right)\right\rangle=\frac{g(u, v)}{\left|x_{12}\right|^{\Delta_{12}^{+}}\left|x_{34}\right|^{\Delta_{34}^{+}}}\left(\frac{\left|x_{24}\right|}{\left|x_{14}\right|}\right)^{\Delta_{12}^{-}}\left(\frac{\left|x_{14}\right|}{\left|x_{13}\right|}\right)^{\Delta_{34}^{-}},
$$

where $\Delta_{i j}^{ \pm}=\Delta_{i} \pm \Delta_{j}, i, j=1,2,3,4$ and $\Delta_{i}$ is the scaling dimension of $\mathcal{O}_{i}$, while $u=\frac{x_{12}^{2} x_{34}^{2}}{x_{13}^{2} x_{24}^{2}}$ and $v=\frac{x_{14}^{2} x_{23}^{2}}{x_{13}^{2} x_{24}^{2}}$ are the cross ratios, we have

$$
g_{p, r}(u, v)=\sum_{h=0}^{r} g_{h}(u, v) \equiv \sum_{h=0}^{r} \frac{p ! r !}{(h !)^{2}} u^{(p+r-2 h) \nu / 2} \sum_{k=0}^{r-h} \frac{v^{-k \nu}}{(k !)^{2}(p-h-k) !(r-h-k) !} .
$$

Each $\mathrm{g}_{h}(u, v)$ can be expanded in terms of conformal blocks $G_{\Delta, \ell}^{a, b}(u, v)$, i.e. eigenfunctions of the quadratic and quartic Casimir invariants of the conformal group. $\Delta$ and $\ell$ are the scaling dimension and the spin of the primaries that could appear in the OPE of $\phi^{p}\left(x_{1}\right) \phi^{r}\left(x_{2}\right)$ and $a=\nu \frac{r-p}{2}, b=-a$. The set of primaries contributing to $\mathrm{g}_{h}(u, v)$ belongs to the linear Regge trajectory $\Delta_{n, \ell}=(p+r-2 h+2 n) \nu+\ell$ :

$$
\mathrm{g}_{h}(u, v)=\sum_{n, \ell}^{\infty} \mathrm{c}_{\phi^{p}}^{2} \phi^{r} \mathcal{O}_{\Delta_{n, \ell}} G_{\Delta_{n, \ell}}^{a, b}(u, v)
$$

Knowing the spectrum of the contributing primaries one may compute term by term the associated OPE coefficients, using for instance the method described in appendix $A$ of [45]. In some cases from the first few OPE coefficients one can infer the general form for any $\ell$ and $n$. In particular if we put $r=1$ in (A.3) we find

$$
g_{p, 1}(u, v)=p u^{\nu(p-1) / 2}+u^{\nu(p+1) / 2}\left(1+\frac{p}{v^{\nu}}\right) .
$$

The first term describes a single primary, as

$$
u^{\nu(p-1) / 2}=G_{\nu(p-1), 0}^{a,-a}(u, v), \quad a=\frac{\nu(1-p)}{2},
$$

while the primaries contributing to the second term belong to the linear Regge trajectory $\Delta_{\ell} \equiv \Delta_{0, \ell}=(p+1) \nu+\ell$. They coincide with the set of spinning operators considered in the present paper. It is not difficult to verify, using the method of [45], that associated OPE coefficients are those given in (3.13). 
In the study of the five-point function we need to compute the OPE coefficient $\mathrm{c}_{\phi^{p} \phi^{p} \mathcal{J}_{\ell}}$, where the conserved HS current $\mathcal{J}_{\ell}$ has scaling dimension $2 \nu+\ell$. This can be extracted from the conformal block expansion of $g_{p, p}(u, v)$, more precisely from the term $\mathrm{g}_{p-1}$ of (A.3). We have

$$
\mathrm{g}_{p-1}(u, v)=p^{2} u^{\nu}\left(1+\frac{1}{v^{\nu}}\right) .
$$

Apart from the $p^{2}$ factor, it coincides with the $g$ function of $\left\langle\phi\left(x_{1}\right) \phi\left(x_{2}\right) \phi\left(x_{3}\right) \phi\left(x_{4}\right)\right\rangle$, therefore we can infer at once eq. (4.13).

Open Access. This article is distributed under the terms of the Creative Commons Attribution License (CC-BY 4.0), which permits any use, distribution and reproduction in any medium, provided the original author(s) and source are credited.

\section{References}

[1] S. Ferrara, A.F. Grillo and R. Gatto, Tensor representations of conformal algebra and conformally covariant operator product expansion, Annals Phys. 76 (1973) 161 [INSPIRE].

[2] A.M. Polyakov, Nonhamiltonian approach to conformal quantum field theory, Zh. Eksp. Teor. Fiz. 66 (1974) 23 [INSPIRE].

[3] A.A. Belavin, A.M. Polyakov and A.B. Zamolodchikov, Infinite conformal symmetry in two-dimensional quantum field theory, Nucl. Phys. B 241 (1984) 333 [InSPIRE].

[4] R. Rattazzi, V.S. Rychkov, E. Tonni and A. Vichi, Bounding scalar operator dimensions in 4D CFT, JHEP 12 (2008) 031 [arXiv:0807.0004] [INSPIRE].

[5] V.S. Rychkov and A. Vichi, Universal constraints on conformal operator dimensions, Phys. Rev. D 80 (2009) 045006 [arXiv:0905.2211] [INSPIRE].

[6] R. Rattazzi, S. Rychkov and A. Vichi, Central charge bounds in $4 D$ conformal field theory, Phys. Rev. D 83 (2011) 046011 [arXiv: 1009.2725] [INSPIRE].

[7] D. Poland and D. Simmons-Duffin, Bounds on $4 D$ conformal and superconformal field theories, JHEP 05 (2011) 017 [arXiv:1009.2087] [InSPIRE].

[8] S. El-Showk et al., Solving the 3D Ising model with the conformal bootstrap, Phys. Rev. D 86 (2012) 025022 [arXiv: 1203.6064] [INSPIRE].

[9] P. Liendo, L. Rastelli and B.C. van Rees, The bootstrap program for boundary CFT $T_{d}$, JHEP 07 (2013) 113 [arXiv:1210.4258] [INSPIRE].

[10] D. Pappadopulo, S. Rychkov, J. Espin and R. Rattazzi, OPE convergence in conformal field theory, Phys. Rev. D 86 (2012) 105043 [arXiv: 1208.6449] [InSPIRE].

[11] S. El-Showk and M.F. Paulos, Bootstrapping conformal field theories with the extremal functional method, Phys. Rev. Lett. 111 (2013) 241601 [arXiv:1211.2810] [INSPIRE].

[12] F. Gliozzi, More constraining conformal bootstrap, Phys. Rev. Lett. 111 (2013) 161602 [arXiv: 1307.3111] [INSPIRE].

[13] D. Gaiotto, D. Mazac and M.F. Paulos, Bootstrapping the 3d Ising twist defect, JHEP 03 (2014) 100 [arXiv:1310.5078] [INSPIRE].

[14] S. El-Showk et al., Solving the $3 d$ Ising model with the conformal bootstrap II. c-minimization and precise critical exponents, J. Stat. Phys. 157 (2014) 869 [arXiv:1403.4545] [INSPIRE]. 
[15] C. Beem, L. Rastelli and B.C. van Rees, The $\mathcal{N}=4$ superconformal bootstrap, Phys. Rev. Lett. 111 (2013) 071601 [arXiv: 1304.1803] [INSPIRE].

[16] Y. Nakayama and T. Ohtsuki, Five dimensional $O(N)$-symmetric CFTs from conformal bootstrap, Phys. Lett. B 734 (2014) 193 [arXiv:1404.5201] [INSPIRE].

[17] Y. Nakayama and T. Ohtsuki, Bootstrapping phase transitions in QCD and frustrated spin systems, Phys. Rev. D 91 (2015) 021901 [arXiv:1407.6195] [INSPIRE].

[18] F. Gliozzi and A. Rago, Critical exponents of the $3 d$ Ising and related models from Conformal Bootstrap, JHEP 10 (2014) 042 [arXiv: 1403.6003] [INSPIRE].

[19] S.M. Chester, J. Lee, S.S. Pufu and R. Yacoby, The $\mathcal{N}=8$ superconformal bootstrap in three dimensions, JHEP 09 (2014) 143 [arXiv:1406.4814] [INSPIRE].

[20] F. Kos, D. Poland and D. Simmons-Duffin, Bootstrapping mixed correlators in the $3 D$ Ising model, JHEP 11 (2014) 109 [arXiv: 1406.4858] [inSPIRE].

[21] S.M. Chester, S.S. Pufu and R. Yacoby, Bootstrapping $O(N)$ vector models in $4<d<6$, Phys. Rev. D 91 (2015) 086014 [arXiv:1412.7746] [InSPIRE].

[22] C. Beem, M. Lemos, P. Liendo, L. Rastelli and B.C. van Rees, The $\mathcal{N}=2$ superconformal bootstrap, JHEP 03 (2016) 183 [arXiv:1412.7541] [INSPIRE].

[23] D. Simmons-Duffin, A semidefinite program solver for the conformal bootstrap, JHEP 06 (2015) 174 [arXiv: 1502.02033] [INSPIRE].

[24] N. Bobev, S. El-Showk, D. Mazac and M.F. Paulos, Bootstrapping the three-dimensional supersymmetric Ising model, Phys. Rev. Lett. 115 (2015) 051601 [arXiv:1502.04124] [INSPIRE].

[25] F. Kos, D. Poland, D. Simmons-Duffin and A. Vichi, Bootstrapping the $O(N)$ archipelago, JHEP 11 (2015) 106 [arXiv: 1504.07997] [INSPIRE].

[26] N. Bobev, S. El-Showk, D. Mazac and M.F. Paulos, Bootstrapping SCFTs with four supercharges, JHEP 08 (2015) 142 [arXiv:1503.02081] [INSPIRE].

[27] F. Gliozzi, P. Liendo, M. Meineri and A. Rago, Boundary and interface CFTs from the conformal bootstrap, JHEP 05 (2015) 036 [arXiv: 1502.07217] [INSPIRE].

[28] C. Beem, M. Lemos, L. Rastelli and B.C. van Rees, The $(2,0)$ superconformal bootstrap, Phys. Rev. D 93 (2016) 025016 [arXiv:1507.05637] [INSPIRE].

[29] Y. Nakayama and T. Ohtsuki, Conformal bootstrap dashing hopes of emergent symmetry, Phys. Rev. Lett. 117 (2016) 131601 [arXiv:1602.07295] [InSPIRE].

[30] F. Kos, D. Poland, D. Simmons-Duffin and A. Vichi, Precision islands in the Ising and $O(N)$ models, JHEP 08 (2016) 036 [arXiv:1603.04436] [INSPIRE].

[31] Y. Nakayama, Bootstrapping critical Ising model on three-dimensional real projective space, Phys. Rev. Lett. 116 (2016) 141602 [arXiv:1601.06851] [INSPIRE].

[32] F. Gliozzi, Truncatable bootstrap equations in algebraic form and critical surface exponents, JHEP 10 (2016) 037 [arXiv: 1605.04175] [INSPIRE].

[33] I. Esterlis, A.L. Fitzpatrick and D. Ramirez, Closure of the operator product expansion in the non-unitary bootstrap, JHEP 11 (2016) 030 [arXiv:1606.07458] [INSPIRE].

[34] S. El-Showk and M.F. Paulos, Extremal bootstrapping: go with the flow, arXiv:1605.08087 [INSPIRE]. 
[35] S. Hikami, Conformal bootstrap analysis for Yang-Lee edge singularity, arXiv:1707.04813 [INSPIRE].

[36] W. Li, Inverse bootstrapping conformal field theories, JHEP 01 (2018) 077 [arXiv: 1706. 04054] [INSPIRE].

[37] K.G. Wilson and J.B. Kogut, The renormalization group and the $\epsilon$-expansion, Phys. Rept. 12 (1974) 75 [INSPIRE].

[38] S. Rychkov and Z.M. Tan, The $\epsilon$-expansion from conformal field theory, J. Phys. A 48 (2015) 29FT01 [arXiv:1505.00963] [INSPIRE].

[39] P. Basu and C. Krishnan, $\epsilon$-expansions near three dimensions from conformal field theory, JHEP 11 (2015) 040 [arXiv: 1506.06616] [INSPIRE].

[40] K. Nii, Classical equation of motion and anomalous dimensions at leading order, JHEP 07 (2016) 107 [arXiv: 1605.08868] [INSPIRE].

[41] C. Hasegawa and Yu. Nakayama, $\epsilon$-expansion in critical $\phi^{3}$-theory on real projective space from conformal field theory, Mod. Phys. Lett. A 32 (2017) 1750045 [arXiv:1611.06373] [INSPIRE].

[42] V. Bashmakov, M. Bertolini and H. Raj, Broken current anomalous dimensions, conformal manifolds and renormalization group flows, Phys. Rev. D 95 (2017) 066011 [arXiv: 1609.09820] [INSPIRE].

[43] F. Gliozzi, A. Guerrieri, A.C. Petkou and C. Wen, Generalized Wilson-Fisher critical points from the conformal operator product expansion, Phys. Rev. Lett. 118 (2017) 061601 [arXiv: 1611.10344] [INSPIRE].

[44] P. Liendo, Revisiting the dilatation operator of the Wilson-Fisher fixed point, Nucl. Phys. B 920 (2017) 368 [arXiv: 1701.04830] [INSPIRE].

[45] F. Gliozzi, A.L. Guerrieri, A.C. Petkou and C. Wen, The analytic structure of conformal blocks and the generalized Wilson-Fisher fixed points, JHEP 04 (2017) 056 [arXiv: 1702.03938] [INSPIRE].

[46] A. Codello, M. Safari, G.P. Vacca and O. Zanusso, Leading CFT constraints on multi-critical models in $d>2$, JHEP 04 (2017) 127 [arXiv:1703.04830] [INSPIRE].

[47] J.A. Gracey, Renormalization of scalar field theories in rational spacetime dimensions, arXiv: 1703.09685 [INSPIRE].

[48] R. Gopakumar, A. Kaviraj, K. Sen and A. Sinha, Conformal bootstrap in Mellin space, Phys. Rev. Lett. 118 (2017) 081601 [arXiv:1609.00572] [INSPIRE].

[49] R. Gopakumar, A. Kaviraj, K. Sen and A. Sinha, A Mellin space approach to the conformal bootstrap, JHEP 05 (2017) 027 [arXiv: 1611.08407] [INSPIRE].

[50] P. Dey, A. Kaviraj and A. Sinha, Mellin space bootstrap for global symmetry, JHEP 07 (2017) 019 [arXiv: 1612.05032] [INSPIRE].

[51] P. Dey and A. Kaviraj, Towards a bootstrap approach to higher orders of $\epsilon$-expansion, arXiv: 1711.01173 [INSPIRE].

[52] E.D. Skvortsov, On (un)broken higher-spin symmetry in vector models, arXiv:1512.05994 [INSPIRE].

[53] S. Giombi and V. Kirilin, Anomalous dimensions in CFT with weakly broken higher spin symmetry, JHEP 11 (2016) 068 [arXiv:1601.01310] [INSPIRE]. 
[54] S. Hellerman, D. Orlando, S. Reffert and M. Watanabe, On the CFT operator spectrum at large global charge, JHEP 12 (2015) 071 [arXiv: 1505.01537] [INSPIRE].

[55] L.F. Alday, Large spin perturbation theory for conformal field theories, Phys. Rev. Lett. 119 (2017) 111601 [arXiv:1611.01500] [INSPIRE].

[56] L.F. Alday, Solving CFTs with weakly broken higher spin symmetry, JHEP 10 (2017) 161 [arXiv: 1612.00696] [INSPIRE].

[57] J. Penedones, E. Trevisani and M. Yamazaki, Recursion relations for conformal blocks, JHEP 09 (2016) 070 [arXiv: 1509.00428] [INSPIRE].

[58] S. Weinberg, Minimal fields of canonical dimensionality are free, Phys. Rev. D 86 (2012) 105015 [arXiv: 1210.3864 ] [INSPIRE].

[59] M.S. Costa, J. Penedones, D. Poland and S. Rychkov, Spinning conformal correlators, JHEP 11 (2011) 071 [arXiv: 1107.3554] [INSPIRE].

[60] V.K. Dobrev et al., On the Clebsch-Gordan expansion for the Lorentz group in $n$ dimensions, Rept. Math. Phys. 9 (1976) 219 [InSPIRE].

[61] S.K. Kehrein and F. Wegner, The structure of the spectrum of anomalous dimensions in the $N$ vector model in $(4-\epsilon)$-dimensions, Nucl. Phys. B 424 (1994) 521 [hep-th/9405123] [INSPIRE].

[62] S. Caron-Huot, Analyticity in spin in conformal theories, JHEP 09 (2017) 078 [arXiv: 1703.00278] [INSPIRE].

[63] D. Simmons-Duffin, D. Stanford and E. Witten, A spacetime derivation of the Lorentzian OPE inversion formula, arXiv:1711.03816 [INSPIRE]. 